Supplement of Hydrol. Earth Syst. Sci., 23, 1103-1112, 2019

https://doi.org/10.5194/hess-23-1103-2019-supplement

(c) Author(s) 2019. This work is distributed under

the Creative Commons Attribution 4.0 License.

(c) (1)
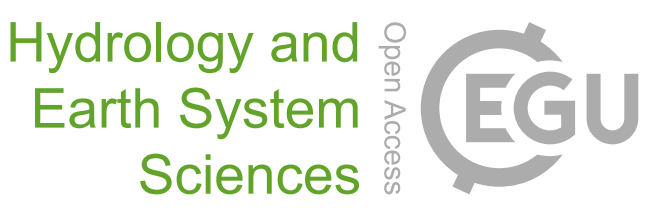

Supplement of

\title{
Technical note: Analytical sensitivity analysis and uncertainty estimation of baseflow index calculated by a two-component hydrograph separation method with conductivity as a tracer
}

Weifei Yang et al.

Correspondence to: Changlai Xiao (xc12822@126.com)

The copyright of individual parts of the supplement might differ from the CC BY 4.0 License. 


\section{Supplement S1}

To verify the mutual offset of random errors, the study collected the daily average conductivity and daily average streamflow of the surface water station with the USGS site number 0297100 (Table 1) from 2001 to 2010 (2979 days in total). Then, office Excel was used to generate 10 sets (2979 per set) of random numbers between -0.05 and 0.05 that obey normal distribution and uniform distribution respectively to simulate the errors (\%) of the daily average conductivity. And 10 sets (2979 per set) of random numbers obeying normal distribution and uniform distribution between -0.05 and 0.05 , respectively, were used to simulate the errors (\%) of the daily average streamflow. Finally, according to different time series (n) (e.g. $30,60,90,120,150,180,210,240,270,300,365,730,1095, \ldots, 2979$, days) sum the errors value ( $\sum_{k=1}^{n} \Delta S C_{k}$ and $\sum_{k=1}^{n} \Delta y_{k}$ ) and analyze the trend of the relative error (\%) of $\sum_{k=1}^{n} S C_{k}$ and $\sum_{k=1}^{n} y_{k}$ with n.

The trend of the average error (\%) of conductivity with $\mathrm{n}$ is shown in Fig. S1. The average errors of the uniform distribution (Fig. S1(a)) and the normal distribution (Fig. S1(b)) are all gradually approach zero with the increase of the time series (n), and the uniform distribution converges faster than the normal distribution. The average errors of the two distributions are between $-2 \%$ and $2 \%$, and the absolute value of the average errors are less than $0.49 \%$ when $\mathrm{n}$ is greater than 365 .

Similar to the conductivity, the trend of the average error (\%) of the streamflow with $\mathrm{n}$ is shown in Fig. S2. The average errors of the uniform distribution (Fig. S2(a)) and the normal distribution (Fig. S2(b)) all gradually approach to zero as the time series (n) increases, and the uniform distribution converges faster than the normal distribution. The average errors of different $n$ under the two distributions are between $-2 \%$ and $2 \%$, and the absolute value of the average errors are less than $0.16 \%$ when $\mathrm{n}$ is greater than 365 .

From the above analysis, when the time series (n) is greater than 365 days ( 1 year), $\Delta_{\sum_{k=1}^{n} s C_{k}}$ BFI will be less than $0.0049 \%$ (0.01 times $0.49 \%$ ), and $\Delta_{\sum_{k=1}^{n} y_{k}}$ BFI will be much less than $0.16 \%$ ( 1 times $0.16 \%$ ). Therefore, the random errors of daily average conductivity and streamflow have a negligible effect on BFI. 


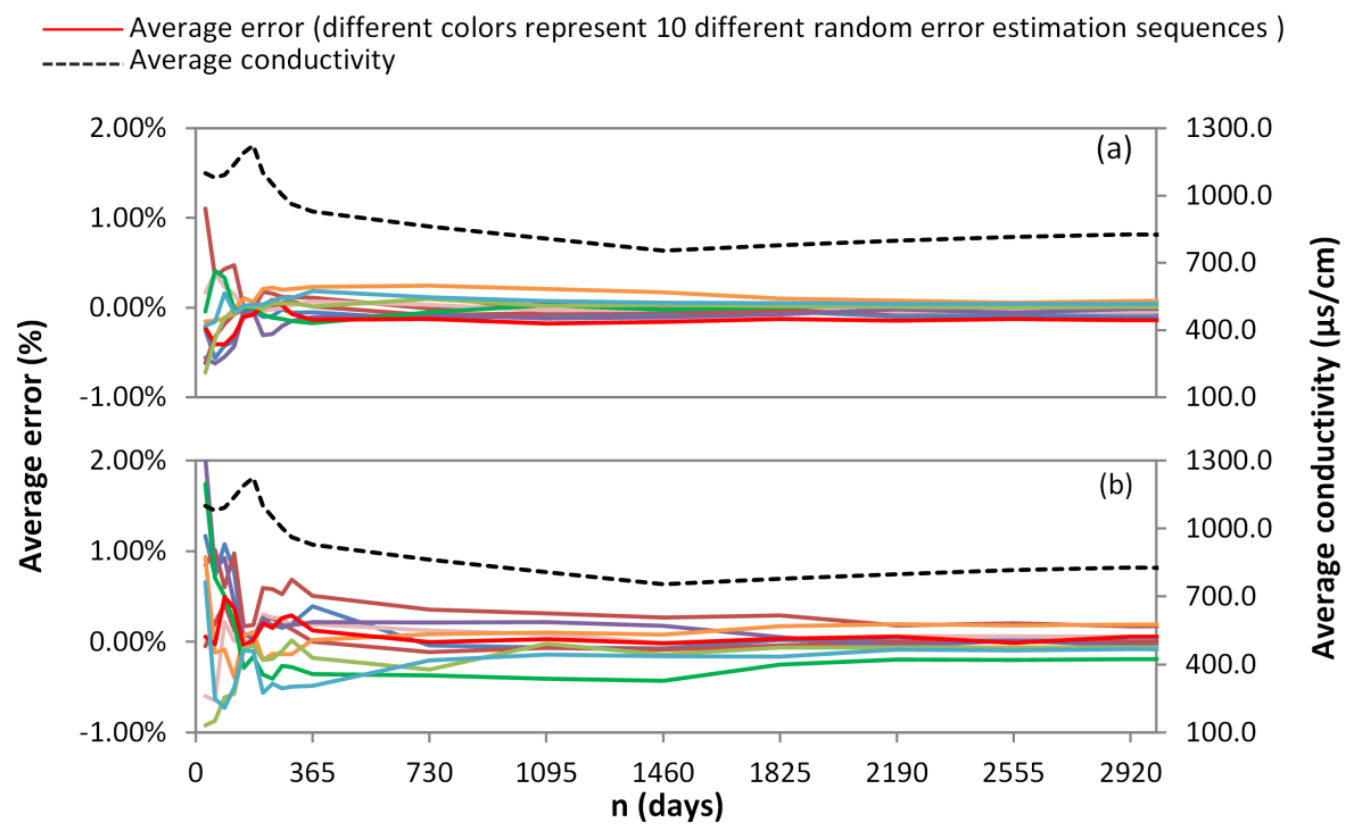

Figure S1. Average conductivity error (\%) with different distributions along the time series (n), (a) uniform distribution, (b) normal distribution.

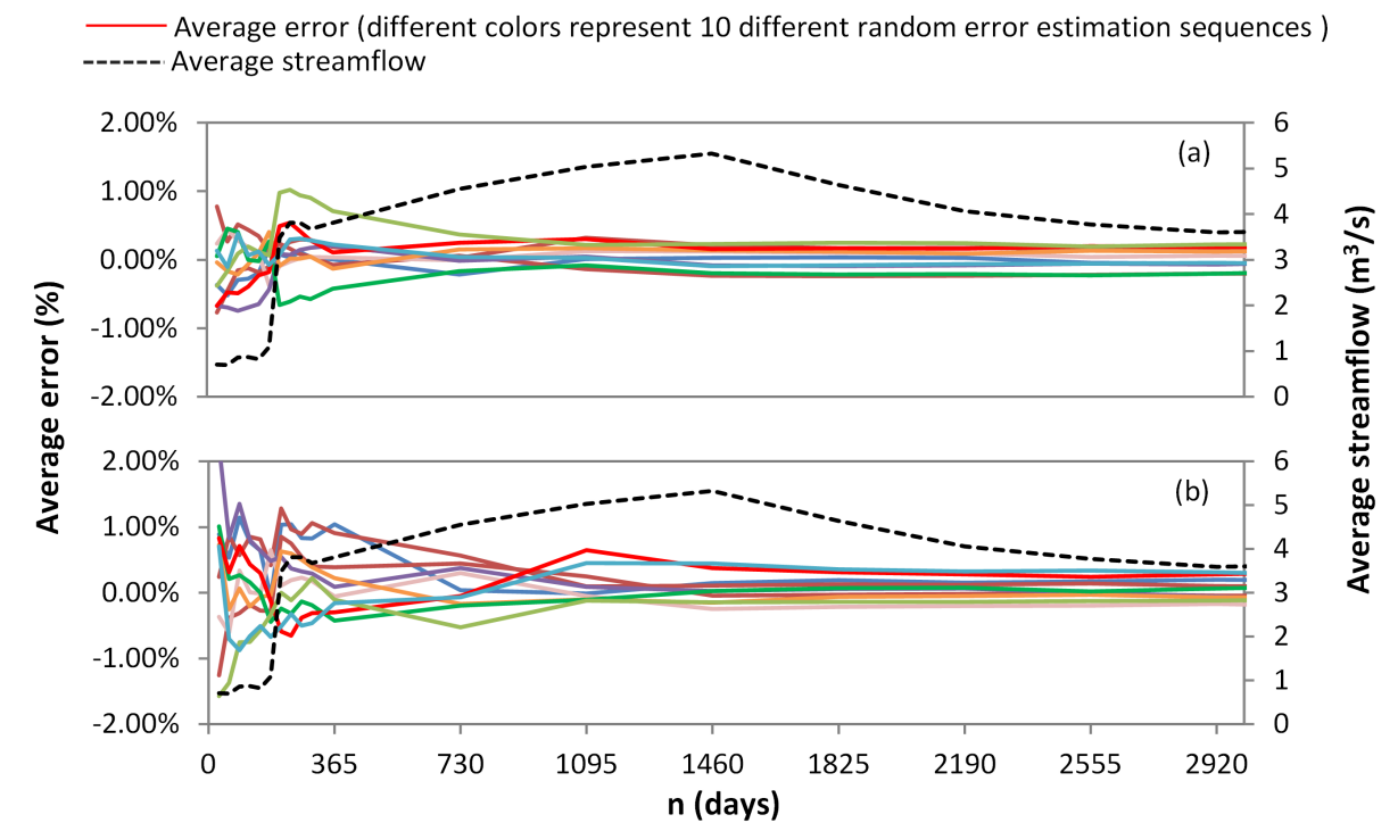

Figure S2. Average streamflow error (\%) with different distributions along the time series (n), (a) uniform distribution, (b) normal distribution. 